\title{
Parameters of Autonomic Nervous System Dysfunction in Acute and Chronic Forms of 1 schemic Heart Disease
}

DOI: $10.17691 /$ stm2017.9.2.21

Received December 23, 2015

A.S. Akulova, PhD Student, Department of Laser Systems and Biomedical Engineering";

A.A. Fedotov, PhD, Associate Professor, Department of Laser Systems and Biomedical Engineering";

K.M. Kolmakova, Physician, Cardiological Unit²;

A.1. Gracheva, Physician, Unit of Functional Diagnosis;

P.A. Lebedev, MD, DSC, Professor, Head of the Therapy Department ${ }^{3}$

(c)

Samara State Aerospace University, 34 Moskovskoye shosse, Samara, 443086, Russian Federation;

2Samara Regional Clinical Hospital named after V.D. Seredavin, 159 Tashkentskaya St., Samara, 443095,

Russian Federation;

3 Samara State Medical University, 89 Chapayevskaya St., Samara, 443099, Russian Federation

The aim of the investigation was to determine the clinical significance of novel parameters of heart rate variability in patients with acute myocardial infarction and chronic ischemic heart disease.

Materials and Methods. The study included 83 male patients, who were divided into three groups: group 1 comprised 30 people with the diagnosis of acute coronary syndrome resulted in myocardial infarction; group 2 consisted of 30 patients with chronic forms of ischemic heart disease; control group 3 included 23 apparently healthy persons without any changes in ECG. ECG signal was recorded using certified computer-based EC-01 electrocardiograph (Novye Pribory, Samara). These records were processed using MATLAB applied program package. The following heart rate variability indices of ECG monitoring at rest and during exercise (6-minute walk test) were analyzed: SDNN, RMSSD, LF/HF ratio, fractal exponent $\alpha$, the total spectral power, acceleration/deceleration (AC/DC) capacity of the heart rate.

Results. The 6-minute walk test leads to unidirectional heart rate variability reduction in both healthy persons and patients with cardiac diseases. As a result, differences in heart rate variability indices between the groups, which were observed at rest, disappear during exercise, except for significantly reduced SDNN and total spectral power value in patients with acute myocardial infarction. A decrease in fractal properties of the heart rate was revealed in patients with coronary artery disease and myocardial infarction on short-term ECG recordings (fractal exponent $\alpha$ ), which was independent of physical activity.

Conclusion. When studying the dysfunction of the autonomic nervous system, new parameters reflecting deceleration (DC) and acceleration $(\mathrm{AC}$ ) of heart rate variability at rest, fractal exponent $\alpha$ independent of physical activity, as well as SDNN and the total spectrum power should be considered along with the classical RMSSD index.

Key words: autonomic nervous system dysfunction; heart rate variability; ischemic heart disease; acute myocardial infarction.

Heart rate variability (HRV) is a known tool of studying autonomic nervous system dysfunction. Alterations of cardiac cycle from contraction to contraction are considered to reflect the balance between sympathetic and parasympathetic influences on the heart, the latter being assigned a cardioprotective role [1]. Acute myocardial infarction (AMI), unstable angina are the most studied forms of ischemic heart disease (IHD), in which evidence of HRV interrelation with clinical prognosis of both sudden death [2] and progression of chronic heart failure has been obtained [3].

The aim of the investigation was to determine the clinical significance of heart rate variability parameters in patients with acute myocardial infarction and chronic ischemic heart disease (CIHD). For this purpose it is necessary to compare classic HRV parameters with those characterizing the symmetry of variability (coefficients of acceleration and deceleration, AC and $D C$ ) and the degree of heart rate fractality (fractal exponent $\alpha$ ) in patients with $\mathrm{AMI}$ and $\mathrm{CIHD}$ at rest; to examine the effect of exercise (6-minute walk test) on the R-R interval variability indices, HRV acceleration and deceleration, as well as the degree of fractality in the groups of patients with CIHD, AMI and the control group.

Materials and Methods. The study included 83 male patients, who were divided into three groups. Group 1 comprised 30 people with AMI (21 patients with Q-wave, 9 patients without Q-wave). Group 2 consisted of 30 patients with various forms of CIHD (18 men with a verified diagnosis of unstable angina pectoris in the stabilization phase, 12 patients with stable exertional

For contacts: Petr A. Lebedev, e-mail: lebedcard@rambler.ru 
angina, I $\square$ II functional class) admitted to the cardiological unit of Samara Regional Clinical Hospital named after V.D. Seredavin. Control group 3 included 23 practically healthy patients having no complaints and ECG changes.

The study was carried out in Samara Regional Clinical Hospital named after V.D. Seredavin in compliance with the Declaration of Helsinki (the Declaration was passed in June 1964, Helsinki, Finland and revised in October 2000, Edinburgh, Scotland) and was performed following approval by the Ethic Committee of the hospital. All examined patients were informed on the content and aims of the study and gave their written informed consent to participate in it.

All patients were measured body mass, height, and arterial pressure prior to the study.

Criteria for inclusion were: chronic heart failure higher than functional class II, arterial hypertension higher than grade II, chronic kidney disease higher than stage II, chronic obstructive pulmonary disease grade II and higher, hemoglobin index in the blood below $110 \mathrm{~g} / \mathrm{L}$, any heart rate disturbances including atrioventricular blockages higher than grade I, frequent extrasystole (more than 30 per hour).

All patients were examined before the discharge from the hospital, but not earlier than 10 days after admission.

In CIHD group, the average age was $59.3 \pm 11.5$ years, in AMl group $59.5 \pm 8.4$ years. The groups were comparable by body mass index: $28.26 \pm 3.94$ and $28.14 \pm 3.57 \mathrm{~kg} / \mathrm{m}^{2}$, respectively. Left ventricular ejection fraction parameter showed the absence of systolic dysfunction and was equal in CIHD group to $58.13 \pm 8.65 \%$, in AMl group to $56.1 \pm 7.55 \%$. There were no significant differences in the parameters of lipid profile, glycemia, renal filtering function (Table 1). Concentration of blood troponin and creatine phosphokinase in group 1 (See Table 1) reflects necrosis of cardiomyocytes, confirming AMI in all patients of this group.

In the control group, the average age was statistically significantly less and amounted to $49.5 \pm 4.6$ years.

ECG signal was recorded from standard lead II at rest with the following 6-minute walk test. ECG recording was performed using a certified computer-based EC-01 electrocardiograph (Novye Pribory, Russia) having standard technical characteristics: a pass band by the level of $\square 3 \mathrm{~dB}$ was $0.05 \square 100 \mathrm{~Hz}$; sampling rate $1,000 \mathrm{~Hz}$; $A D$ conversion capacity 16 bit. Computer processing of ECG signal including stages of digital signal filtration, detection of characteristic points, formation of $R-R$ interval duration set and determining HRV parameters was done by the MATLAB R2013a software package for engineering computations.

Spectral and frequency parameters of ECG monitoring were evaluated during the investigation (SDNN, RMSSD, high-frequency (HF) and low-frequency (LF) components, fractal exponent $\alpha$, total spectral power TP, AC/DC) at rest and during exercise.

SDNN is a standard deviation of NN interval (R-R interval) length (ms):

$$
\operatorname{SDNN}=\sqrt{\frac{1}{N} \sum_{i=1}^{N}\left(X_{i}-\bar{X}\right)^{2}},
$$

where $X_{i}$ is the value of i-th $\mathrm{R}-\mathrm{R}$ interval; $\bar{X}$ is a mean value of $\mathrm{R}-\mathrm{R}$ interval length; $N$ is the size of the tested $\mathrm{R}-\mathrm{R}$ sample.

A statistical parameter RMSSD is the square root of the squares of successive differences between adjacent $\mathrm{R}-\mathrm{R}$ intervals (ms):

$$
\text { RMSSD }=\sqrt{\frac{1}{N-1} \sum_{i=1}^{N-1}\left(X_{i+1}-\bar{X}\right)^{2}},
$$

where $\bar{X}_{i}$ is the value of $\mathrm{i}$-th $\mathrm{R}-\mathrm{R}$ interval; $\bar{X}_{i+1}$ is the value of $(i+1)$-th $R-R$ interval length; $N$ is the size of R-R sample.

Fractal exponent $\boldsymbol{\alpha}$ is determined using detrended fluctuation analysis (DFA) [4]. DFA method allows the study of the structure of various processes, including non-stationary ones, from the point of view of statistical self-similarity.

The algorithm of computing the fractal exponent $\alpha$ is as follows [4]. In the first stage, a cumulative sum $W_{t}$ is formed from the time succession of $X \mathrm{R}-\mathrm{R}$ interval lengths:

$$
W_{t}=\sum_{i=1}^{t} X_{i}
$$

where $X_{i}$ is the value of $\mathrm{i}$-th $\mathrm{R}-\mathrm{R}$ interval; $t$ is a variable changing from 1 to the value of $X R-R$ interval initial sample size.

In the second stage, the cumulative sum $W_{t}$ is divided into time windows $L$ of equal size; interpolation polynomial

Table 1

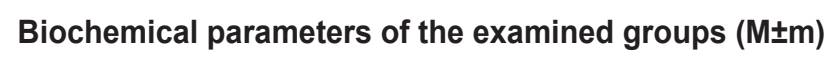

\begin{tabular}{lcc}
\hline \multicolumn{1}{c}{ Parameters } & $\begin{array}{c}\text { Group 1, } \\
\text { acute myocardial } \\
\text { infarction }(\mathrm{n}=30)\end{array}$ & $\begin{array}{c}\text { Group 2, } \\
\text { chronic ischemic } \\
\text { heart disease }(\mathrm{n}=30)\end{array}$ \\
\hline Total cholesterol $(\mathrm{mmol} / \mathrm{L})$ & $5.33 \pm 1.24$ & $4.85 \pm 1.18$ \\
\hline $\mathrm{LDL}(\mathrm{mmol} / \mathrm{L})$ & $3.58 \pm 0.98$ & $3.42 \pm 1.18$ \\
\hline $\mathrm{HDL}(\mathrm{mmo} / \mathrm{L})$ & $1.17 \pm 0.26$ & $1.11 \pm 0.32$ \\
\hline Triglycerides $(\mathrm{mmol} / \mathrm{L})$ & $1.77 \pm 1.21$ & $1.77 \pm 1.49$ \\
\hline Troponin I $(\mathrm{ng} / \mathrm{ml})$ & $26.29 \pm 35.82$ & - \\
\hline Creatine phosphokinase-MB $(\mathrm{ng} / \mathrm{mL})$ & $90.73 \pm 13.59^{*}$ & $15.46 \pm 4.45$ \\
\hline Glucose $(\mathrm{mmo} / \mathrm{L})$ & $8.47 \pm 4.48$ & $6.69 \pm 1.37$ \\
\hline Creatinine $(\mu \mathrm{mmol} / \mathrm{L})$ & $100.64 \pm 19.90$ & $102.48 \pm 21.53$ \\
\hline Glomerular filtration rate, MDRD & & \\
$\left.(\mathrm{ml} / \mathrm{min} / 1.73 \mathrm{~m})^{2}\right)$ & $64.17 \pm 14.92$ & $64.44 \pm 17.10$ \\
\hline
\end{tabular}

${ }^{*} p<0.01$ compared to chronic ischemic heart disease group. 
is formed for each time window, in case of using the firstorder DFA method it is a linear polynomial $Z$.

In the third stage, a mean square deviation $F$ is calculated for each time window $L$ :

$$
F=\left[\frac{1}{L} \sum_{j=1}^{L}\left(X_{t j}-Z_{j}\right)^{2}\right]^{0.5} .
$$

In the fourth stage, computations 2 and 3 are repeated over a range of different time window lengths $L$.

Finally, the first-order fractal exponent $\alpha$ is determined as a relation of $\log F$ fluctuations as a function of $L$ change.

Coefficients of acceleration and deceleration ( $A C$ and $D C$ ) of the heart rate are very sensitive to determination of the sudden cardiac mortality risk and are significant predictors of patient survival after myocardial infarction [5]. These parameters are non-linear and define the intensity of quasi-periodical trends in the heart rate on the basis of phase-rectified signal averaging [6].

The coefficients of acceleration and deceleration of the heart rate are determined in the following way. First, anchor knots of acceleration and deceleration are isolated from $\mathrm{R}-\mathrm{R}$ length series. $\mathrm{R}-\mathrm{R}$ interval is considered an anchor point of deceleration, if the value of its length exceeds the length of the previous $R-R$ interval. $\mathrm{R}-\mathrm{R}$ interval is an anchor point of acceleration, if the length of the running $R-R$ interval is less than the length of the previous one. To increase noise immunity in determining $A C / D C$ values, additional conditions may be introduced in this stage, e.g. maximum permissible value may be set between the lengths of two successive $R-R$ intervals.

In the second stage, a supporting fragment in the center of which an anchor point must be located, is formed for each anchor point of acceleration or deceleration from the initial $R-R$ series. The optimal window width of the supporting fragment depends on the initial $\mathrm{R}-\mathrm{R}$ sample and is usually taken within $12 \square 60 \mathrm{R}-\mathrm{R}$ intervals [5].

In the third stage, the formed supporting fragments are averaged separately for all acceleration and deceleration anchor points, as a result, two integral curves are formed

(Figure 1).
Figure 1. Diagrams of variation of heart rate variability indices in the tested groups at rest (blue) and during walking (green):

(1) patients with myocardial infarction; (2) with chronic ischemic heart disease; (3) control group 
And finally, $A C$ and $D C$ coefficients are calculated:

$$
D C(A C)=\frac{Q(0)+Q(1)-Q(-1)-Q(-2)}{4},
$$

where $Q$ is the value of the averaged length of the respective $R-R$ intervals on the acceleration and deceleration integral curve; $Q(0)$ is the averaged length value of all $R-R$ intervals corresponding to the anchor points of acceleration and deceleration.

Results. Values of HRV indices for all examined groups at rest and during 6-minute walk test are presented in Table 2. The results obtained were processed using Statistica 6.0 program for Windows. To assess the significance of the mean values in the compared groups, nonparametric Mann-Whitney U-test was applied, differences were considered statistically significant at $p<0.05$.

Figure 1 shows a box-and-whisker plot, on which the median, $25^{\text {th }}$ and $75^{\text {th }}$ percentiles, as well as maximal and minimal values of HRV indices for all groups are indicated. Sensitivity and specificity of the HRV indices used were assessed according to a binary pathology/ norm scale (Figure 2). For quantitative evaluation of the binary classification quality, the value of the area under the ROC-curve (receiver operating characteristic) was computed for each HRV index: for the fractal exponent $\alpha$ it amounted to 0.845 ; for RMSSD it was 0.857 ; for deceleration

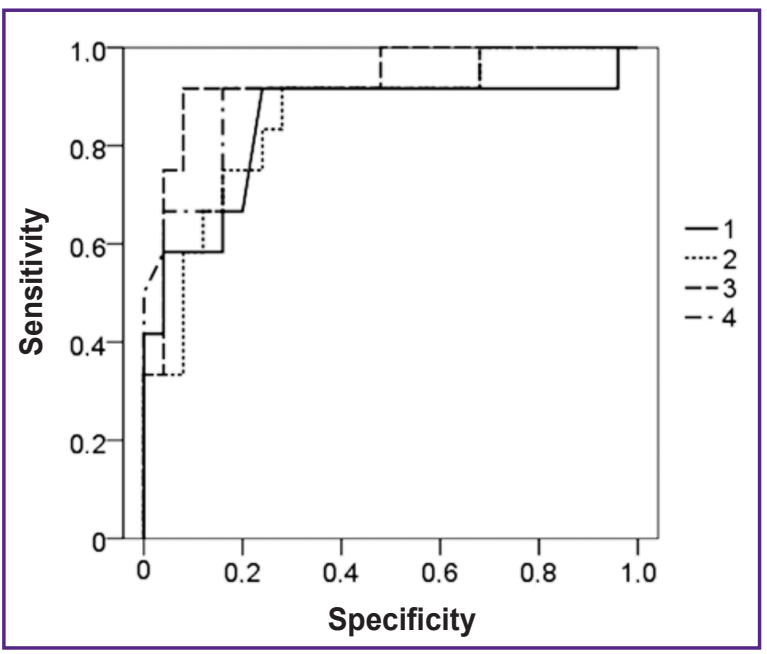

Figure 2. Error curves of binary classification of patient condition using heart rate variability indices:

(1) fractal exponent $\alpha$; (2) RMSSD; (3) coefficient of cardiac rate deceleration DC; (4) coefficient of cardiac rate acceleration $\mathrm{AC}$
Heart rate variability parameters in the examined groups (Me [25\%; 75\%])

\begin{tabular}{|c|c|c|c|}
\hline Parameters & $\begin{array}{c}\text { Group 1, acute } \\
\text { myocardial infarction }\end{array}$ & $\begin{array}{c}\text { Group 2, chronic } \\
\text { ischemic heart disease }\end{array}$ & $\begin{array}{l}\text { Group 3, } \\
\text { control }\end{array}$ \\
\hline $\mathrm{AC}_{1}$ & $-5.80[-8.10 ;-4.05]^{*}$ & $-5.75[-8.15 ;-4.42]^{*}$ & $-14.70[-18.60 ;-9.90]$ \\
\hline $\mathrm{AC}_{2}$ & $-4.75[-3.90 ;-2.55]$ & $-3.60[-4.90 ;-2.60]$ & $-3.20[-5.10 ;-2.55]$ \\
\hline $\mathrm{DC}_{1}$ & $5.50[4.18 ; 7.25]^{*}$ & $5.50[3.90 ; 7.27]^{*}$ & $14.30[11.85 ; 19.75]$ \\
\hline $\mathrm{DC}_{2}$ & $3.80[3.15 ; 5.10]$ & $3.90[2.50 ; 6.30]$ & $3.90[2.60 ; 5.65]$ \\
\hline $\mathrm{SDNN}_{1}$ & $33.50[22.30 ; 39.75]^{*}$ & $28.00[21.50 ; 38.75]^{*}$ & $60.00[41.50 ; 72.50]$ \\
\hline $\mathrm{SDNN}_{2}$ & $22.00[18.50 ; 30.00]^{*+}$ & $30.00[22.00 ; 34.00]$ & $35.00[26.25 ; 38.00]$ \\
\hline $\mathrm{RMSSD}_{1}$ & $20.60[15.65 ; 29.00]^{*}$ & $16.10[10.12 ; 23.80]^{*}$ & $33.0[24.35 ; 42.48]$ \\
\hline $\mathrm{RMSSD}_{2}$ & $16.00[10.80 ; 21.75]^{*}$ & $11.90[8.10 ; 25.60]$ & $9.20[5.40 ; 17.20]$ \\
\hline$a_{1}$ & $0.94[0.84 ; 0.98]^{*}$ & $0.89[0.83 ; 0.96]^{*}$ & $0.82[0.70 ; 0.86]$ \\
\hline$a_{2}$ & $1.01[0.89 ; 1.06]$ & $1.03[0.87 ; 1.16]$ & $0.99[0.94 ; 1.10]$ \\
\hline $\mathrm{TP}_{1}$ & $1,887[945 ; 2,673]^{*}$ & $1,481[912 ; 2,941]^{*}$ & $6,751[3,342 ; 10,005]$ \\
\hline $\mathrm{TP}_{2}$ & $947[687 ; 1,686]^{\star+}$ & $1,712[783 ; 2,267]$ & $2,654[1,426 ; 3,675]$ \\
\hline $\mathrm{LF}_{1} / \mathrm{HF}_{1}$ & $1.50[0.83 ; 2.50]^{*+}$ & $3.15[1.30 ; 5.00]$ & $4.40[3.12 ; 5.42]$ \\
\hline $\mathrm{LF}_{2} / \mathrm{HF}_{2}$ & $1.60[1.00 ; 2.30]^{*}$ & $2.90[1.80 ; 5.00]^{*}$ & $5.20[3.78 ; 7.90]$ \\
\hline $\mathrm{HR}_{1}$ & $61.00[57.00 ; 67.00]$ & $65.00[57.00 ; 74.75]$ & $72.00[65.00 ; 76.75]$ \\
\hline $\mathrm{HR}_{2}$ & $72.00[67.25 ; 81.25]^{*}$ & $72.00[62.00 ; 86.50]^{*}$ & 102.00 [93.00; 112.50] \\
\hline
\end{tabular}

Note. Index 1: measurement of parameters at rest; index 2: during exercise; * statistically significant differences of values compared to the control group ( $p<0.05) ;{ }^{+}$compared to group 1 and group $2(p<0.05)$.

coefficient DC -0.930 ; for acceleration coefficient AC -0.898 .

Discussion. Analyzing frequency and spectral parameters of ECG R-R intervals at rest, statistically significant changes are noted in SDNN, RMSSD in both groups with IHD, which is in compliance with a classical picture [1 $\square 3$ ]: in acute and chronic forms of IHD HRV decreases. Decrease of general HRV (shown by SDNN) coincides with the reduction of the total spectral power TP and the power of all TP components including a highfrequency constituent HF. As high frequencies of R-R interval variations are associated with the respiration rate and therefore with the periodical excitation of the vagus, it was not surprising that RMSSD, being the variability of the successive interval difference and reflecting their fast changes, also responds by a decrease. These data agree also with the results previously obtained by us [7], which signifies the loss of reflexogenic influences of the parasympathetic system on the sinus node. There is another explanation to the HRV reduction with the increase of the heart rate [8]. According to a simple mathematical rule, one and the same variability requires greater changes of intervals at a slow rate than at a frequent one. Therefore, normalization of HRV to heart rate is necessary to separate physiological influence from purely mathematical relationship.

Reduction of HRV is known to be a significant 
risk factor for patients suffered myocardial infarction. Phase signal averaging makes it possible to isolate the periodicity from the time segments of the record, which can contain unsteady noises and artifacts as well as periodic components with the exclusion of nonperiodic components. And here it is possible to isolate the coefficient of deceleration DC, which describes an averaged characteristic of cardiac rate deceleration caused by modulations of a vagal tone.

Investigations by Bauer et al. [5] showed that the capacity to rate deceleration has a greater clinical importance than the capacity to rate acceleration. Of the total number of patients with early infarction they separated patients which composed $15 \%$ of all examined patients, who had the DC parameter asymmetrically decreased, i.e. much more than AC. This prospective study demonstrated that a marked reduction of the rate deceleration has the greatest prognostic value in revealing patients with the risk of sudden arrhythmic death in comparison with the reduction of general variability according to SDNN and decrease of the coefficient of acceleration. It gives grounds to speak on different mechanisms responsible for acceleration and deceleration of the rhythm. In our work, there was no evident asymmetry of these parameters in al examined patients, i.e. coefficients of acceleration and deceleration were substantially lower in the groups with IHD compared to the control group reflecting a marked decrease of general HRV.

During exercise (6-minute walk test), HRV substantially drops in all examined groups, and all parameters change unidirectionally though the degree of HRV change in the control group is greater than in the groups with IHD. As a result, statistical differences disappear over the majority of the parameters. Evidently, it is related to the increase of sympathetic influences during exercise, which is a proven mechanism [9]. An essential decrease of the total spectral power TP should be noted in patients with AMI during exercise, which determined a statistical difference from the CIHD group by the similar parameter, whereas the groups did not differ at rest.

Of special attention is a different degree of RMSSD and SDNN reduction in the patient groups during exercise. In patients with IHD the general HRV drop was accompanied by a slight RMSSD reduction compared to the parameters at rest, whereas in healthy patients RMSSD decreased so prominently that it became less in the absolute value than in the IHD groups. This speaks about the fact that fast HRV changes during exercise are not typical for healthy people. Capacity to acceleration and deceleration of the heart rate in the examined groups regularly symmetrically decreased, being similar in the mean values $\left(\mathrm{AC}_{2}\right.$ and $\left.\mathrm{DC}_{2}\right)$ in the groups.

A great methodological difficulty of HRV assessment is connected with unstable character of R-R intervals. Heart rate fluctuations possess the property of time selfsimilarity (fractal characteristics), therefore long-term ECG records presented by Holter monitoring are mainly used.
In our work, 6-minute fragments of ECG records have been analyzed. Presence of long-term fractal episodes suggests that HRV changes occur not only under the action of a momentary cause but are explained by a specific memory. Reduction of fractality degree of the heart rate is noted in chronic heart failure [10]. The investigation [8] showed that non-linear HRV analysis can surpass the classical analysis methods in their capacity to predict a sudden cardiac death in patients with arrhythmogenic substrate, for example, after myocardial infarction. The fractal exponent $\alpha$ has been found not to change, whereas HRV determined by the classical methods decreases [11]. This is confirmed by the findings of our work.

Periodic HRV components are traditionally evaluated using a frequency analysis [1]. High frequencies are believed to reflect the parasympathetic tone while low frequencies reflect the sympathetic tone. Therefore, the LF/HF ratio reflects the balance of the autonomic nervous system. Perine et al. [9] examined these parameters in healthy people during exercise, at a controlled respiration, and changes of body position. The authors believe that spectral parameters do not reflect increase of sympathetic and decrease of parasympathetic contribution during exercise. The power of HF spectrum is noted over the whole range of exercises, and during intensive exercises causes the peak values of HRV power. The power of LF-spectrum does not change during a low-intensity exercise in a sitting position but drops essentially when the load increases demonstrating a full inconsistency with the increase of the sympathetic activity during exercise. Meanwhile, statistically significant differences of value in the level of fitness and age in the examined patients were not found. LF power grew substantially at moderately intensive exercise in a lying position. Different mechanisms of arterial pressure control are likely to be involved in these situations. LF are known to be partly caused by the activity of baroreflex when the rate of cardiac contraction is decelerated if pressure in the aorta increases [9]. Billman [12] shows in his work that it is impossible to physiologically interpret this common index. There are also difficulties in interpreting the effect of exercise on HRV in sick and healthy people, since its intensity during 6-minute walk test is not standardized.

In the work [13] no dependence of LF/HF on the intensity of exercise was found, whereas SDNN and fractal exponent a regularly decreased proportionally to the force of pedalling when short-term ECG fragments were analyzed. These data reflect contradictions occurring during physiological interpretation of HRV parameters. Some researchers [14] assume that there are differences between the concepts of autonomic nervous system tone and autonomic modulation. The authors think that a simplified interpretation of sympathyvagal balance as a sum of influences on the sinus node of the two branches of autonomic nervous system is untenable. The system of heart rate regulation seems 
to be multilevel and complex, and from this viewpoint, fractal characteristics reflect more adequately regulatory disorders without explaining their mechanisms, though fixing the fact of their impairments.

The investigations, conducted by us, showed that differences of HRV parameters being at rest disappear during 6-minute walk test, which results in unidirectional decrease of HRV both in sick and healthy. However, in patients with AIM, SDNN and the total spectral power decrease significantly.

Variability related to the acceleration of the heart rate pace and its deceleration in IHD changes mainly symmetrically. And in this case general HRV in patients with IHD is considerably lower relative to the control group.

It has been estimated that decrease of the cardiac rate fractality degree in patients with IHD on the shortterm fragments of ECD record (fractal exponent $\alpha$ ) does not depend on exercise.

In our work, there was demonstrated a comparable specificity of new parameters of nonlinear dynamics of cardiac rate $(\alpha, A C, D C)$ in comparison with the classical parameters of the statistical analysis in detecting autonomic dysfunction in IHD patients. In this context, absence of close correlations of the fractal exponent a with HRV parameters in patients with $\mathrm{CIHD}$ and $\mathrm{AMI}$ signifies a potential clinical usefulness of this parameter. Absence of considerable differences in HRV between the groups with AMI and CIHD is likely to be explained by the fact that current methods of treating patients with AMI have a great clinical efficacy, diminishing the size of necrosis, a morphological substrate of arrhythmogenicity and chronic heart failure [15]. As a result, the parameters of autonomic nervous system dysfunction in AMI approximate the parameters of patients with chronic forms of IHD.

Conclusion. When studying the dysfunction of the autonomic nervous system, it is necessary to take into consideration the parameters characterizing the symmetry of variability at rest in patients with acute and chronic forms of ischemic heart disease, the fractal exponent a independent of physical activity, as well as SDNN and the total spectrum power in patients with acute myocardial infarction along with the classical heart rate variability indices.

Study Funding. The work was supported by the Ministry of Education and Science of the Russian Federation within the project part of the governmental task in the sphere of scientific activity (project code 12.2013.2014/K, state registration number 11421670017).

Conflicts of Interest. The authors have no conflicts of interest related to the investigation.

\section{References}

1. Schwartz P.J., La Rovere M.T., Vanoli E. Autonomic nervous system and sudden cardiac death. Experimental basis and clinical observations for post-myocardial infarction risk stratification. Circulation 1992; 85(1 Suppl): $177-191$.
2. Kiviniemi A.M., Tulppo M.P., Wichterle D., Hautala A.J., Tiinanen S., Seppänen T. Mäkikallio T.H., Huikuri H.V. Novel spectral indexes of heart rate variability as predictors of sudden and non-sudden cardiac death after an acute myocardial infarction. Ann Med 2007; 39(1): 54-62, https://doi. org/10.1080/07853890600990375.

3. Shah S.A., Kambur T., Chan C., Herrington D.M., Liu K., Shah S.J. Relation of short-term heart rate variability to incident heart failure (from the Multi-Ethnic Study of Atherosclerosis). Am J Cardiol 2013; 112(4): 533-540, https://doi.org/10.1016/j. amjcard.2013.04.018.

4. Peng C.K., Buldyrev S.V., Havlin S., Simons M., Stanley H.E., Goldberger A.L. Mosaic organization of DNA nucleotides. Phys Rev E 1994; 49(2): 1685-1689, https://doi. org/10.1103/physreve.49.1685.

5. Bauer A., Kantelhardt J.W., Barthel P., Schneider R., Mäkikallio T., Ulm K., Hnatkova K., Schömig A., Huikuri H., Bunde A., Malik M., Schmidt G. Deceleration capacity of heart rate as a predictor of mortality after myocardial infarction: cohort study. Lancet 2006; 367(9523): 1674-1681, https://doi. org/10.1016/s0140-6736(06)68735-7.

6. Bauer A., Kantelhardt J.W., Bunde A., Barthel P., Schneider R., Malik M., Schmidt G. Phase-rectified signal averaging detects quasi-periodicities in non-stationary data. Physica A: Statistical Mechanics and its Applications 2006; 364: 423-434, http://dx.doi.org/10.1016/j.physa.2005.08.080.

7. Povalyaeva R.A., Lebedev P.A., Kalakutsky L.I., Bubnova M.G., Aronov D.M. Computerised photoplethysmography in assessment of myocardial infarct rehabilitation efficiency. Kardiosomatika 2012; 3(2): 5-10.

8. Huikuri H.V., Stein P.K. Heart rate variability in risk stratification of cardiac patients. Prog Cardiovasc Dis 2013; 56(2): 153-159, https://doi.org/10.1016/j.pcad.2013.07.003.

9. Perini R., Veicsteinas A. Heart rate variability and autonomic activity at rest and during exercise in various physiological conditions. Eur J Appl Physiol 2003; 90(3-4): 317-325, https://doi.org/10.1007/s00421-003-0953-9.

10. Peng C.K., Havlin S., Stanley H.E., Goldberger A.L. Quantification of scaling exponents and crossover phenomena in nonstationary heartbeat time series. Chaos 1995; 5(1): 82 87, https://doi.org/10.1063/1.166141.

11. Weippert M., Behrens K., Rieger A., Kumar M., Behrens M. Effects of breathing patterns and light exercise on linear and nonlinear heart rate variability. Appl Physiol Nutr Metab 2015; 40(8): 762-768, https://doi.org/10.1139/apnm2014-0493.

12. Billman G.E. The LF/HF ratio does not acurately measure cardiac sympatho-vagal balance. Front Phy siol 2013; 4, https://doi.org/10.3389/fphys.2013.00026.

13. Chen S.W., Liaw J.W., Chang Y.J. Chuang L.L., Chien C.T. Combined heart rate variability and dynamic measures for quantitatively characterizing the cardiac stress status during cycling exercise. Comput Biol Med 2015; 63: 133-142, https://doi.org/10.1016/j.compbiomed.2015.05.026.

14. Nicolini P., Ciulla M.M., De Asmundis C.. Magrini F., Brugada $P$. The prognostic value of heart rate variability in the elderly, changing the perspective. from sympathovagal balance to chaos theory. Pacing Clin Electrophysiol 2012, 35(5): 622-638, https.//loi.org/10.1111/j.15408159,2012.03335.x.

15. Huikuri H.V., Stein P.K. Clinical application of heart rate variability after acute myocardial infarction. Front Physiol 2012; 3, https://doi.org/10.3389/fphys.2012.00041. 